\title{
All-Optical Ternary Stability in Uniform Nonlinear Chalcogenide Fiber Bragg Gratings
}

\author{
Elham Yousefi ${ }^{a, b}$, Mohsen Hatamic ${ }^{\text {, Amin Torabi Jahromid }}{ }^{\text {, and Sajjad Dehghani }}{ }^{\mathrm{e}}$ \\ aFaculty of Science, Persian Gulf University of Bushehr, Bushehr 7516913817, Iran. \\ ${ }^{b}$ Atomic and Molecular Group, Faculty of Physics, Yazd University, Yazd, Iran. \\ cPhysics Department, Shiraz University of Technology, Shiraz, Iran. \\ dElectrical Engineering Department, Persian Gulf University of Bushehr, Bushehr \\ 7516913817, Iran. \\ eFaculty of Advanced Technologies, Shiraz University, Shiraz, Iran.
}

Corresponding author' Email: sdehghani@shirazu.ac.ir

Received: Jan. 2, 2018, Revised: Feb. 12, 2018, Accepted: May. 8, 2018, Available Online: Dec. 27, 2019

DOI: 10.29252/ijop.13.2.103

\begin{abstract}
In recent decades, fiber Bragg gratings (FBGs) have been very much considered for their many applications in optical communication systems, as well as due to their bistability and multi stability properties. In this paper, the formation of ternary stability (TS) in nonlinear chalcogenide fiber Bragg gratings (NCFBGs) is investigated via numerical simulations. Effective parameters on TS such as the FBG length, input wavelength and nonlinear property (or nonlinearity) on TS formation are introduced and studied. It is found that there exists a minimum length for each third order nonlinear coefficient that TS phenomena can be observed. Also, the threshold intensity for TS formation is calculated with respect to the length, input wavelength and third order nonlinearity. In addition, the relevance between the minimum length for TS formation and the third order nonlinearity in the range of chalcogenide nonlinearities are looked into. It is numerically confirmed that increasing the input wavelength (in a valid FBG input wavelength range) increases the TS formation threshold intensity, while decreases the needed FBG length. Because of using experimental values in this paper, it has valuable information about designing the alloptical device with three - level stability which makes NCFBG a suitable option for all-optical ternary switching and all-optical memory in the integrated optical circuits.
\end{abstract}

KEYWORDS: All-Optical devices, Chalcogenide fiber Bragg grating, Multistabiltiy, Nonlinear optics.

\section{I.INTRODUCTION}

Today fiber Bragg gratings (FBGs), because of the having shining features on optical communication systems, are highly considered all over the scientific and industrial world. Their various applications are known in the linear optical communication systems such as pulse dispersion compensation [1], optical filters [2], wavelength division multiplexing (WDM), optical limiters [3], and optical sensors [4]. They are also well-regarded for their nonlinear effects such as optical switching [5] and optical bistability [6, 7] which is applicable in all-optical memories and all-optical signal processing devices which has become a significant interest for research recently [8-10]. Chalcogenide glasses offer such opportunity because of their nonlinear parameter, $\mathrm{n}_{2}$, which is about 100 to 1000 times larger compared with silica [11-14]. This type of FBGs shows bistability characteristics $[15,16]$ in suitable conditions as well as multistability characteristics like ternary stability (TS) which provides a good platform for speeding up the information transfer rate compared to that of the binary logic [17]. 
In the present study, we consider a uniform nonlinear chalcogenide glass [10] made FBG taking into account its third order nonlinearity. We solve the governing coupled mode equations numerically and find special initial values to obtain TS as a kind of multistability. After that, we study this TS diagram which has three stable branches in input-output intensity diagram and introduce its characteristics. Finally, we investigate the effects of some important parameters like: FBG length, input wavelength $\left(\lambda_{0}\right)$ and third order nonlinearity on the TS formation. This paper is a part of the first leading work on three level stabilities in FBG to design the suitable FBG for different applications in all-optical ternary memory and integrated optical circuits.

\section{THEORY AND NUMERICAL Calculations}

The schematic diagram of a typical lossless uniform FBG with length $L$ is shown in Fig. 1. Its refractive index varies along the length periodically and can be formulated as follows [8]:

$$
n(z)=n_{0}+n_{1} \cos \left(\frac{2 \pi}{\Lambda} z\right)+n_{2}|E|^{2}
$$

where $E$ is the electric field in the FBG, $\Lambda$ is the grating period, $n_{0}, n_{1}$ and $n_{2}$ denote the linear refractive index, refractive index modulation amplitude and third order nonlinear refractive index coefficient, respectively.

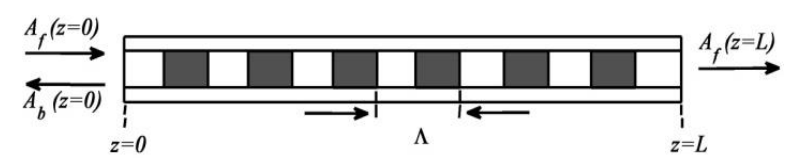

Fig. 1. The schematic diagram of the FBG with length $L$. The forward and backward waves are shown [10].

The electric field in the FBG can be described as combining the forward and backward waves [8]:

$$
\begin{aligned}
E(\mathbf{r}, t)= & \frac{1}{2} F(x, y)\left[A_{f}(z, t) e^{i \beta_{B} z}\right. \\
& \left.+A_{b}(z, t) e^{-i \beta_{B} z}\right] e^{-i \omega_{0} t}+c . c .,
\end{aligned}
$$

where $\omega_{0}$ is the carrier frequency, $A_{f}$ and $A_{b}$ represent the slowly varying amplitude of forward and backward waves respectively, and $F(x, y)$ is the transverse variations of two counter propagating waves.

We assume that the incident wave is continuous. Substituting Eqs. (1) and (2) into the Maxwell's equations and using slowly varying amplitude approximation, the nonlinear coupled mode equations for both forward and backward wave amplitudes are obtained in as follows [8]:

$$
\begin{aligned}
\frac{\partial A_{f}}{\partial z}+\frac{\alpha}{2} A_{f} & =i \delta A_{f}+i \kappa A_{b} \\
& +i \gamma\left(\left|A_{f}\right|^{2}+2\left|A_{b}\right|^{2}\right) A_{f}, \\
-\frac{\partial A_{b}}{\partial z}+\frac{\alpha}{2} A_{b} & =i \delta A_{b}+i \kappa A_{f} \\
& +i \gamma\left(\left|A_{b}\right|^{2}+2\left|A_{f}\right|^{2}\right) A_{b},
\end{aligned}
$$

where $\alpha$ is the FBG loss that is neglected, $\delta$, $\kappa$ and $\gamma$ account for the detuning from the Bragg wavelength, coupling coefficient and nonlinear coefficient, respectively. The numerical solution of nonlinear coupled mode equations is derived by using Runge-Kutta methods. We do these calculations for the FBG as it is discussed in $[10,18]$ by doing our simulation from end to the beginning of the FBG with appropriate boundary conditions. It is done by MATLAB software.

\section{OPTICAL TERnARY STABILITY Characteristics}

As shown in Fig. 2, a typical TS diagram is a kind of hysteresis diagram that has five branches; odd branches are stable while even ones are unstable as shown in Fig. 2. To study the characteristics of TS, five parameters are introduced, namely TS interval, first and second on-off switching ratios, and first and 
second TS threshold intensities which are clearly introduced in Fig. 2. Also, the typical data used in this work is shown in Table 1.

Table 1. Typical data which are used in this article.

$$
\begin{array}{ll}
\lambda_{B}=1550 \mathrm{~nm} & n_{0}=2.45 \\
\lambda=1550.015 \mathrm{~nm} & n_{1}=1.5 \times 10^{-4} \\
\Lambda=3.16 \times 10^{-7} \mathrm{~m} & n_{2 \text { silica }}=0.273 \times 10^{-19} \mathrm{~m}^{2} / \mathrm{W}_{\text {[19] }} \\
n_{2}=220 n_{\text {2silica }}[11] &
\end{array}
$$

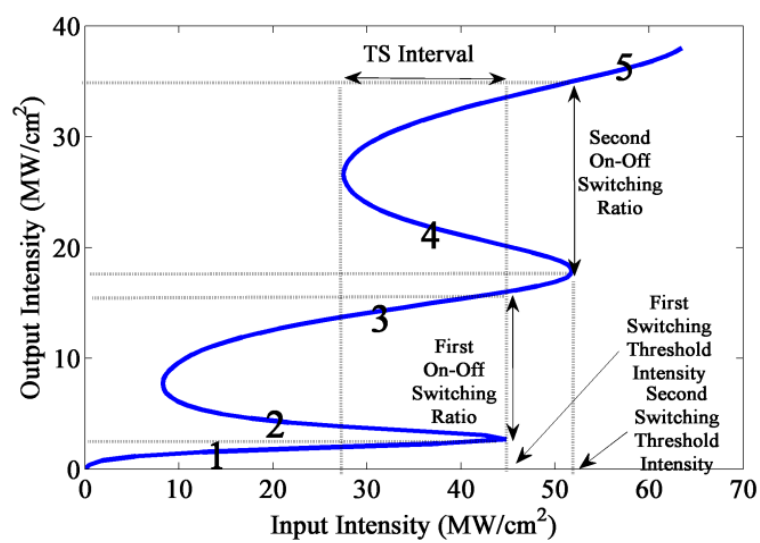

Fig. 2. An illustrative definition of five parameters of TS, first and second on-off switching ratios, first and second switching threshold intensities and TS interval. Five branches of the TS are also clearly depicted.

The two most important parameters which can be criteria for TS formation are: TS interval and second switching threshold intensity. The TS interval is an input intensity overlapping area between branches 1, 3 and 5 in typical TS diagram which is shown in Fig. 2. It means that in TS interval, for each intensity point there are three output points. If the TS diagram has a minimum TS interval that means the TS is formed i.e. with no TS interval there exist no TS curve. Also, it is clear that the second switching threshold intensity is a critical and minimum value of input intensity to form the TS diagram via these characteristics (as shown in Fig. 2). Therefore, the second switching threshold intensity should have certain values for TS formation which is also related to the TS interval. In this state when the TS diagram starts to form, "second switching threshold intensity" can also be called "minimum TS intensity" or "TS formation threshold intensity".
In the next sections, we investigate the minimum values of input intensity for TS formation with considering the effects of FBG length, input intensity, third order nonlinearity and input wavelength. Also it is obtained that by varying those parameters and selecting good values, the TS interval can be reachable.

\section{IV.How IS A TERNARY STABILITY FORMED?}

TS is not obtained in nonlinear chalcogenide FBG unless the necessary conditions are maintained. Parameters like FBG length, third order nonlinearity and input power are important for TS to be observed. Two main parameters which can considerably affect the TS formation for a given nonlinearity are "the FBG length" and "the input pulse intensity".

As it is already known, chalcogenide materials have a range of nonlinearities about 100 to 1000 times of silica glasses. Table 2 shows the linear and third-order nonlinear refractive indices for some of the chalcogenide materials which we have used for simulating the behavior of TS formation.

Table 2 Measured linear refractive index $n_{0}$ and third-order nonlinear refractive index $\mathrm{n}_{2}$ of chalcogenide glasses [11].

\begin{tabular}{lll}
\hline \hline Glass & $\mathbf{n}_{\mathbf{0}}$ at $\mathbf{1 . 5 5} \boldsymbol{\mu \mathbf { m }}$ & $\mathbf{n}_{2} / \mathbf{n}_{\mathbf{2} \text {,ilica }}(\mathbf{\pm 1 5 \% )}$ \\
\hline \hline $\mathrm{As}_{40} \mathrm{~S}_{60}$ & 2.45 & 220 \\
$\mathrm{As}_{40} \mathrm{~S}_{40} \mathrm{Se}_{20}$ & 2.55 & 300 \\
$\mathrm{As}_{40} \mathrm{~S}_{50} \mathrm{Se}_{10}$ & 2.49 & 380 \\
$\mathrm{As}_{40} \mathrm{~S}_{30} \mathrm{Se}_{30}$ & 2.62 & 430 \\
$\mathrm{As}_{25} \mathrm{~S}_{55} \mathrm{Te}_{20}$ & 2.52 & 470 \\
$\mathrm{As}_{40} \mathrm{~S}_{10} \mathrm{Se}_{50}$ & 2.76 & 560 \\
$\mathrm{As}_{30} \mathrm{Se}_{61}$ & 2.81 & 660 \\
$\mathrm{As}_{40} \mathrm{Se}_{55} \mathrm{Cu}_{5}$ & 2.93 & 850 \\
$\mathrm{As}_{40} \mathrm{Se}_{60}$ & 2.81 & 930 \\
\hline
\end{tabular}

\section{THE EFFECT OF FBG LENGTH AND INPUT INTENSITY ON TERNARY STABILITY FORMATION}

First of all, the effect of FBG length and $\kappa L$ (the production of the coupling coefficient, $\kappa$, and the FBG length) on TS formation are studied for each chalcogenide glass third-order nonlinearity. Diagrams shown in Fig. 3 
illustrate these effects on the TS formation for $\mathrm{n}_{2} / \mathrm{n}_{2 \text {, silica }}=220$ but for various values of $\kappa L$.

As shown in Fig. 3 assuming $\kappa L=5$ for simulation, TS is not formed even by increasing the input intensity. Although some individual bistable curves are achieved, there is no TS interval. After that, the value of $\kappa L$ is increased to 7 while keeping other parameters fixed. With these conditions, TS is formed well, as seen in Fig. 3 with a solid curve, such that TS interval has a considerable value.

Other simulations are carried out for various values of $\kappa L$ to obtain the critical one for starting the TS formation i.e. the minimum value of $\kappa L$ required for TS formation. The dashed curve in Fig. 3 depicts the critical situation for starting the TS formation (in fact, TS interval starts to construct), by taking $\kappa L=5.3, \quad \mathrm{n}_{2} / \mathrm{n}_{2 \text {,silica }}=220, \quad$ and other parameters are held fixed.

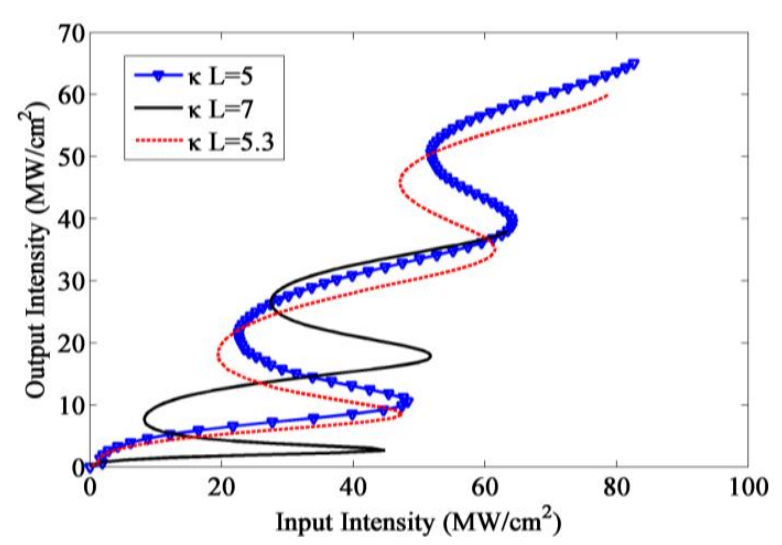

Fig. 3. The input-output diagrams for $\mathrm{n}_{2} / \mathrm{n}_{2 \text {, silica }}=220$ and three different $\kappa L$.

In the next step of our simulations, $\kappa L$ is fixed to 5.2 (a little lower the critical $\kappa L$ ) while the value of third order nonlinearity is varied. We want to see if the increasing on third-order nonlinearity can compensate the lack of value of $\kappa L$ or not.

The results of simulations for three values of third-order nonlinearities chosen from Table 2 are presented in Fig. 4. Dashed diagram illustrates the input-output intensity diagram for $\mathrm{n}_{2} / \mathrm{n}_{2, \text { silica }}=300$. It shows that although TS is near to threshold formation, TS is not yet formed. It is expected that by increasing the value of third-order nonlinearity, the TS can be reached. However, as shown in Fig. 4 for $\mathrm{n}_{2} / \mathrm{n}_{2, \text { silica }}=660 \mathrm{TS}$ is not formed yet and even for the maximum value of $n_{2} / n_{2 \text {,silica }}$ i.e. 930 , unpredictably TS is not formed as well.

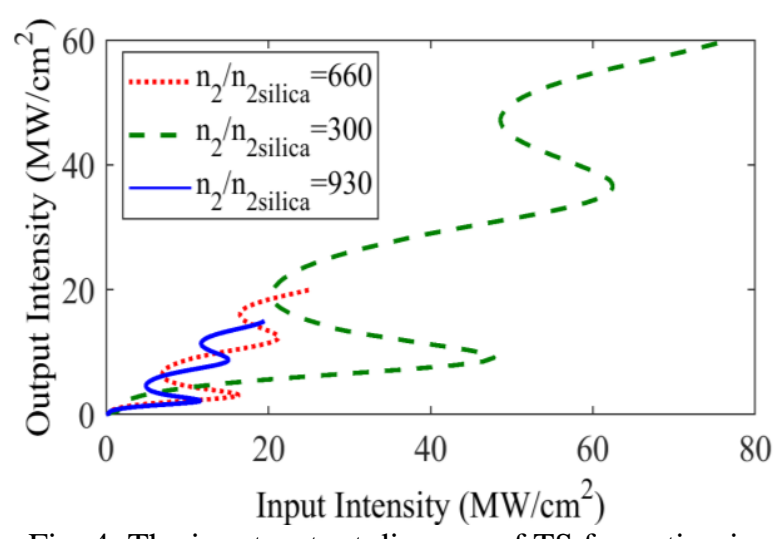

Fig. 4. The input-output diagram of TS formation in $\kappa L=5.2$ and various $\mathrm{n}_{2} / \mathrm{n}_{2, \text { silica }}$.

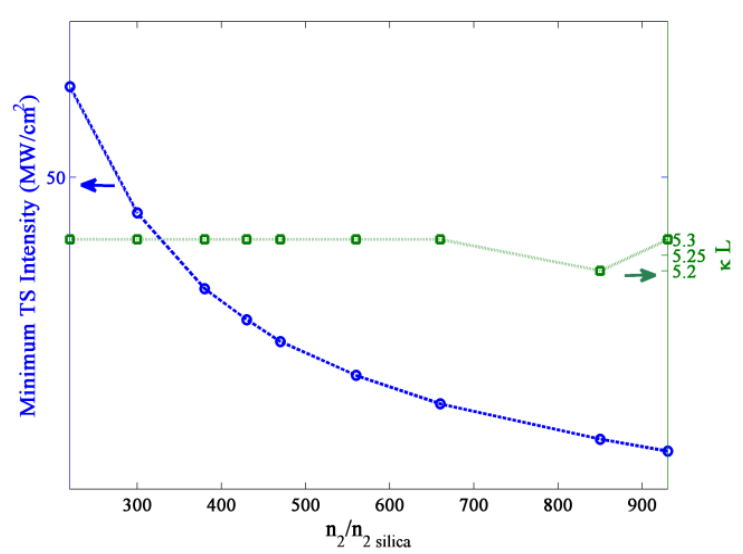

Fig. 5. The minimum intensities in the range of about $10-70 \mathrm{MW} / \mathrm{cm}^{2}$ and the minimum $\kappa L$ (that is about 5.3) needed for TS formation in several chalcogenide third order nonlinearities.

In continue, the minimum $\kappa L$ for the TS formation is calculated for various chalcogenide third-order nonlinearities. The result is depicted in Fig. 5. It is worth to mention that the minimum necessary FBG length of TS formation is nearly constant (that its value is about $1.7 \mathrm{~cm}$ ) and independent of the third-order nonlinearity. Having mentioned that the minimum input intensity, i.e. the second switching threshold intensity, which is needed for TS formation is decreased from $62 \mathrm{MW} / \mathrm{cm}^{2}$ to $15 \mathrm{MW} / \mathrm{cm}^{2}$ by increasing the 
nonlinearity (i.e. the volume of $n_{2} / n_{2, \text { silica }}$ from 220 to 930), as expected. Also Fig. 5 presents such behaviors. In Fig. 6, all results are compared to show the effect of minimum FBG length and minimum intensity for TS formation for different values of chalcogenide nonlinearities.

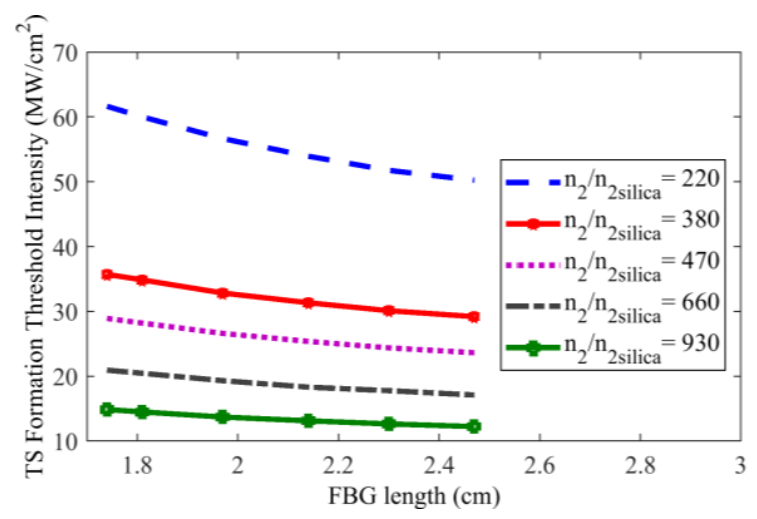

Fig. 6. Diagram of TS formation threshold intensity versus the FBG length in various third order nonlinearity for $\lambda_{0}=1550.015 \mathrm{~nm}$.

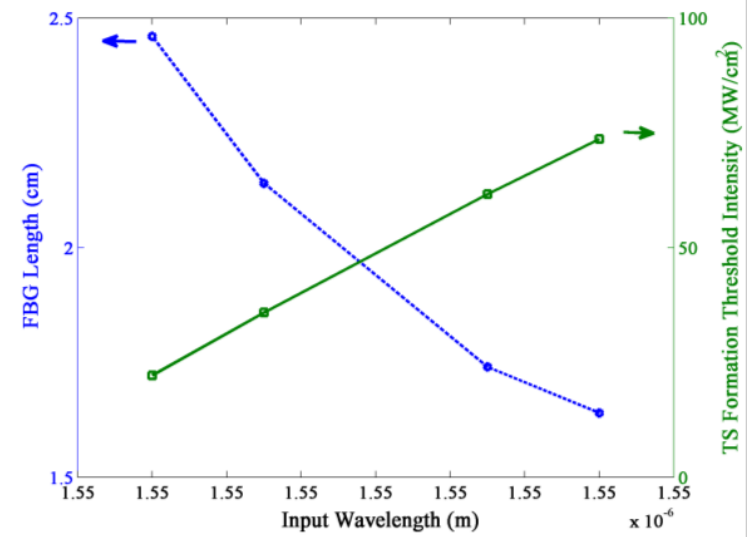

Fig. 7. The minimum length of FBG and the minimum intensity for TS formation in various input wavelengths.

\section{CONCLUSION}

In this paper, the performance of the FBG is simulated by numerically solving the governing nonlinear coupled mode equations to study the ternary stability (TS). First, the special initial values are obtained in which output-input intensity diagram shows TS. Also, five parameters that represent the characteristics of TS are introduced. Usually bistability is formed in nonlinear FBGs but TS formation needs special conditions. Our simulation is conducted for the chalcogenide materials made FBG with third-order nonlinearity in the range of about 100-1000 times of the silica one. The Bragg wavelength is $1550 \mathrm{~nm}$. We showed that the minimum FBG length for the TS formation is nearly constant (about $1.7 \mathrm{~cm}$ ) and nearly independent of the third-order nonlinearity for the range of chalcogenide nonlinearities. We obtained minimum necessary intensities for each chalcogenide type for the TS formation with respect to the FBG length. There is a minimum length below which, TS will not be formed even by increasing the nonlinearity. It is also illustrated that by increasing the input wavelength in a valid range of input wavelengths, the required TS formation intensities will drop while the minimum FBG length will increase. It is worth to mention that TS can be formed in chalcogenide glasses of a few centimeters length which makes it a good alternative for all-optical ternary memory in the integrated optical circuits.

\section{REFERENCES}

[1] A.B. Dar and R.K. Jha, "Chromatic dispersing compensation techniques and characterization of fiber Bragg grating for dispersion compensation," Opt. Quant. Electron. Vol. 49, p. 108, 2017.

[2] M.M. Ali, K.S. Lim, A. Becir, M.H. Lai, and H. Ahmad, "Optical Gaussian Notch Filter Based on Periodic Microbent Fiber Bragg Grating," IEEE Photon. J. Vol. 6, pp. 1-8, 2014.

[3] X. Han and J. Yao, "Bandstop-to-Bandpass Microwave Photonic Filter Using a PhaseShifted Fiber Bragg Grating," Journal of Lightwave Technology, Vol. 33, pp. 51335139, 2015.

[4] G. Alvarez-Botero, F.E. Baron, C.C. Cano, O. Sosa, and M. Varon, "Optical sensing using fiber Bragg gratings: fundamentals and applications," IEEE Instrument Measurement Mag. Vol. 20, pp. 33-38, 2017.

[5] E. Yousefi, M. Hatami, and S. Dehghani, "Optimization of Bistability in nonlinear chalcogenide fiber Bragg grating for all optical switch and memory applications," Int. J. Opt. Photon. Vol. 11, pp. 49-55, 2017. 
[6] H.G. Winful, J.H. Marburger, and E. Garmire, "Theory of Bistability in Nonlinear Distributed feedback structures," Appl. Phys. Lett. Vol. 35, pp. 379-381, 1979.

[7] N.G.R. Broderick, "Bistable switching and multiple gap-soliton formation in a fiber Bragg grating," Opt. Commun. Vol. 148, pp. 90-94, 1998.

[8] G.P. Agrawal, Applications of Nonlinear Fiber Optics, Academic Press, 2001.

[9] Y.L. Kim, J.H. Kim, S. Lee, D.H. Woo, S.H. Kim, and T.H. Yoon, "Broad-band all optical flip-flop based on optical bistability in an integrated SOA/DFB-SOA," IEEE Photon. Technol. Lett. Vol. 16, pp. 398-400 , 2004.

[10]E. Yousefi and M. Hatami, "All optical self signal processing using chalcogenide nonlinear fiber Bragg grating,". Optik, Vol. 125, pp. 6637-6640, 2014.

[11] J.M. Harbold, F.O. Ilday, and F.W. Wise, "Highly nonlinear As-S-Se glasses for alloptical switching," Opt. Lett. Vol. 27, pp. 119121, 2002.

[12] M.R. Lamont, B. Luther-Davies, D.Y. Choi, S. Madden, and B.J. Eggleton, "Supercontnuum generation in dispersion engineered highly nonlinear $(\gamma=10 / \mathrm{M} / \mathrm{w}) \quad \mathrm{As}_{2} \mathrm{~S}_{3}$ chalcogenide planar waveguide," Opt. Express, Vol.16, pp. 14938-14944, 2008.

[13]L. Scholtz and J. Müllerová, "Numerical studies on wavelength-selective all-optical switching using optical bistability in nonlinear chalcogenide FBGs," ICTON, Budapest, Vol. 39, pp. 1-4, 2015.

[14]Z. Tahmasebi and M. Hatami, "Study of the gain saturation effect on the propagation of dark soliton in $\mathrm{ER}^{+3}$-doped $\mathrm{Ga}_{5} \mathrm{Ge}_{20} \mathrm{Sb}_{10} \mathrm{~S}_{65}$ chalcogenide fiber amplifier," Opt. Commun. Vol. 284, pp. 656-659, 2011.

[15] Y. Yosia and S. Ping, "Optical bistability in periodic media with third fifth- and seventhorder nonlinearities," J. Lightwave Technol. Vol. 25, pp. 875-882, 2007.

[16] Y. Yosia and S. Ping, "Double Optical Bistability and its Application in Nonlinear Chalcogenide-Fiber Bragg Gratings,' Phys. B, Vol. 394, pp. 293-296, 2007.

[17]E. Yousefi, M. Hatami, and A. Torabi Jahromi, "All-optical ternary signal processing using uniform nonlinear chalcogenide fiber
Bragg gratings," J. Opt. Soc. Am. B, Vol. 32, pp. 1472-1478, 2015.

[18] A.G.F. Filho, J.R.R. Sousa, A.F. Morais Neto, J.W.M. Menezes, and A.S.B. Sombra, "Periodic modulation of nonlinearity in a fiber Bragg grating: a numerical investigation," J. Electromagn. Analysis Appl. Vol. 04, pp. 5356, 2012.

[19]G.P. Agrawal, Nonlinear Fiber Optics, Academic Press, 2013.

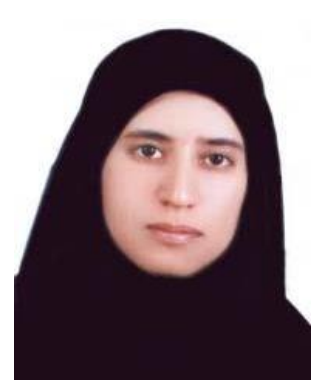

Elham Yousefi was born in Shiraz, Iran. She received her $\mathrm{BSc}, \mathrm{MSc}$, and $\mathrm{PhD}$ in Atomic and Molecular Physics from Qom University, Alzahra University and Yazd Unversity in 2004, 2010 and 2015, respectively. Her main research interests include: Nonlinear Optics, Multi-level Integrated Optics and Solar cells.

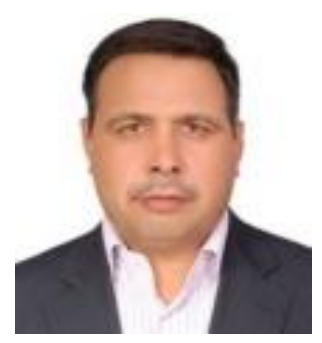

Mohsen Hatami was born in Abadeh, Iran. $\mathrm{He}$ received his $\mathrm{BSc}, \mathrm{MSc}$, and $\mathrm{PhD}$ degrees in Nuclear Physics, Theoretical Physics and Atomic Physics (Optics and Lasers) from Shiraz University, Shiraz, Iran in 1990, 1993 and 2006, respectively. He became an associate professor in 2011 at Yazd University. From 2008 to 2012 he was the head of Atomic and Molecular Physics at Yazd University. Now he is an academic staff in Shiraz University of Technology. His current research interests include: Nonlinear Optics, Optical Soliton, Integrated Optics, and Photonics. Dr. Hatami is a member of the 
Optics and Photonics Society of Iran (OPSI) and Physics Society of Iran.

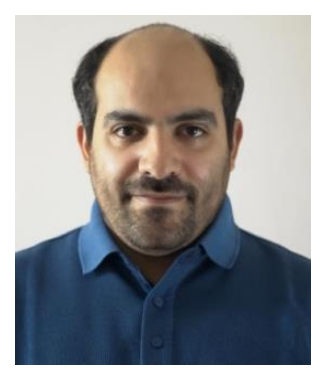

Amin Torabi Jahromi received his BSc and MSc degrees from Shiraz University, Iran, majoring in control engineering in 2004 and 2007, respectively. He received his $\mathrm{PhD}$ from Nanyang Technological University of Singapore in 2014 and currently serves as an assistant professor in Persian Gulf University of Bushehr, Iran. He worked on many practical projects and industrial challenges during his academic studies and also supervised many undergraduate and graduate students in Student Research Center of Shiraz University, Radio Amatory student research center at Shiraz Engineering College and also Singapore Institute of Manufacturing Technology. During his $\mathrm{PhD}$ studies, he worked with an award winning team that secured the IES Prestigious Engineering Achievement Award 2011.

His research interests include fault tolerant systems, neuro-fuzzy and dynamic fuzzy neural networks, nonlinear and adaptive control systems, digital control systems, clustering algorithms, data analysis and data mining and their applications in various control, mechanical and telecommunication systems. Besides, he is also interested in implementation of above mentioned techniques on embedded systems using microprocessor and microcontrollers as well as FPGAs. Iurl $\{$ http://www.torabi-amin.ir $\}$

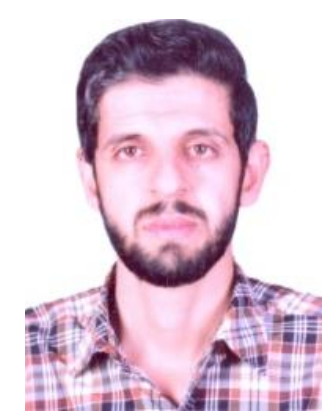

Sajjad Dehghani received his BSc and MSc degrees from Shiraz University and PhD from Tarbiat Modares University in Electronic Engineering, in 2002, 2006 and 2012, respectively. He is an assistant professor in Electronic Engineering at Shiraz University, Shiraz, Iran. His current research interests include: Nanoelectronics, Optoelectronics, Thin film Solar cells, Gas sensors, Nanocoatings. 
THIS PAGE IS INTENTIONALLY LEFT BLANK. 